\title{
An error-based video quality assessment method with temporal information
}

\author{
Woei-Tan Loh ${ }^{1}$ • David Boon Liang Bong ${ }^{1}$
}

Received: 26 July 2017 / Revised: 27 March 2018 / Accepted: 8 May 2018 /

Published online: 1 June 2018

(C) Springer Science+Business Media, LLC, part of Springer Nature 2018

\begin{abstract}
Videos are amongst the most popular online media for Internet users nowadays. Thus, it is of utmost importance that the videos transmitted through the internet or other transmission media to have a minimal data loss and acceptable visual quality. Video quality assessment (VQA) is a useful tool to determine the quality of a video without human intervention. A new VQA method, termed as Error and Temporal Structural Similarity (EaTSS), is proposed in this paper. EaTSS is based on a combination of error signals, weighted Structural Similarity Index (SSIM) and difference of temporal information. The error signals are used to weight the computed SSIM map and subsequently to compute the quality score. This is a better alternative to the usual SSIM index, in which the quality score is computed as the average of the SSIM map. For the temporal information, the second-order time-differential information are used for quality score computation. From the experiments, EaTSS is found to have competitive performance and faster computational speed compared to other existing VQA algorithms.
\end{abstract}

Keywords Video quality $\cdot$ Temporal effects $\cdot$ Temporal distortions $\cdot$ Multimedia content

\section{Introduction}

Many multimedia applications deal with visual assets nowadays. This is more evident in mobile devices such as smartphones, tablets, smart watches, and smart glasses. These devices use microprocessors or microcontrollers with high processing ability. This processing ability enables them to process data on their own without relying on external processing devices. According to the research done by Ooyala [18], mobile video views increased from $6.3 \%$ of the overall mobile traffic data in 2012 to $45.1 \%$ in Q3 2015. This shows the importance of videos in multimedia equipment. Assessment of the video qualities of these products is crucial as a quality feedback tool for device manufacturers and content service providers.

David Boon Liang Bong

davidblbong@yahoo.com; bbldavid@unimas.my

1 Faculty of Engineering, Universiti Malaysia Sarawak, Kota Samarahan, Malaysia 